\title{
Zum individuellen und kollektiven Umgang mit einer Pandemie (On the individual and collective handling of a pandemic)
}

\author{
Astrid Schreyögg
}

Angenommen: 7. Juli 2021 / Online publiziert: 1. September 2021

(C) Springer Fachmedien Wiesbaden GmbH, ein Teil von Springer Nature 2021

Steven Taylor (2020): Die Pandemie als psychologische Herausforderung. Ansätze für ein psychosoziales Krisenmanagement. Gießen: Psychosozial-Verlag, 185 S., 19,90€ (first published 2019 by Cambridge Scholar Publishing)

Dieses Buch von dem Professor und klinischen Psychologen an der University of British Columbia in Vancouver, Kanada, ist in mehrfacher Hinsicht erstaunlich: Zum einen hat der Autor das Buch geschrieben, bevor noch irgendjemand etwas von der aktuellen Pandemie mit Covid 19 geahnt hat. Seine Intentionen zielten ursprünglich eher auf Pandemien im Zusammenhang mit Varianten der Influenza, d.h. mit Grippeviren. Zum anderen thematisiert Taylor vorrangig psychologische bzw. psychosoziale Phänomene, während wir in der aktuellen Situation viel häufiger und ausführlicher über biologische oder medizinische Besonderheiten informiert wurden.

Das Buch ist in 12 Kapitel gegliedert. Im ersten gibt der Autor einen kursorischen Überblick über Pandemien: Er erläutert den Begriff und beschreibt die bislang bekanntesten Varianten wie die verschiedenen Formen der Influenza, sodann die Beulenpest, HIV, also Aids, Typhus, Sars, Mers usw. Er thematisiert auch, dass es sich bei viralen Pandemien regelmäßig um Zoonosen handelt, also um Krankheiten, die ursprünglich von Tieren auf Menschen übertragen wurden. Ihre Effekte seien allerdings meistens durch zusätzliche bakterielle Infektionen verschärft. Der Verlauf der Krankheiten sei unterschiedlich schnell und die Inkubationszeit unterschiedlich lang. Jede Pandemie erhöhe den individuellen Stress und ziehe Auswirkungen auf das Gesundheitssystem eines Landes und seine gesamte Wirtschaft nach sich. Das Ziel des Buches sieht der Autor in der Vermittlung von Wissen, besonders von psychologischem Wissen, wie Pandemien entstehen, wie ihnen vorzubeugen ist und welche Maßnahmen in einer Pandemie zu ergreifen sind.

Dr. Astrid Schreyögg $(\bowtie)$

Salzachstr. 67, 14129 Berlin, Deutschland

E-Mail: astrid.schreyoegg@googlemail.com 
Im zweiten Kapitel referiert der Autor „moderne Methoden für den Umgang mit Pandemien“. Dazu zählt er öffentliche Aufklärung, also Risiko- und Krisenkommunikation, Informationen über Impfstoffe und antivirale Therapien, über Hygienepraktiken, aber auch über Maßnahmen des Social Distancing. Das Ziel sei immer, den Ausbruch möglichst schnell unter Kontrolle zu bringen. Bei allen diesen Maßnahmen spielten psychologische Faktoren eine zentrale Rolle, denn die Einhaltung oder Nichteinhaltung von Hygieneregeln und Social Distancing seien primär psychologisch determiniert. Deshalb sei vertieftes Wissen von psychologischen Mechanismen zur Bewältigung einer Pandemie ganz zentral.

So geht es im dritten Kapitel um ,psychologische Reaktionen auf Pandemien“. Obwohl manche Menschen mit dem Stress in Pandemien gut zurechtkommen, erzeugt es bei anderen Resignation, bei wieder anderen aber starke Störungen, die bis zu Posttraumatischen Belastungsstörungen reichen. Regelmäßig ließen sich Phantasien über Wunderheilungen beobachten, gelegentlich sogar gesellschaftliche Destabilisierungen mit politischen Exzessen. Bei vielen Menschen führten Pandemien $\mathrm{zu}$ ausgeprägter Angst und sozialer Zerrüttung.

So widmet sich der Autor im vierten Kapitel „Persönlichkeitseigenschaften als Faktoren emotionaler Verletzlichkeit“. Hier ist der Autor nun in seinem eigentlichen Element und referiert mehrere, sich wechselseitig ergänzende Theorie- und Forschungsbereiche, die in einer Pandemie relevant sind. So scheinen auf der einen Seite Personen mit einem unrealistischen Optimismus und dem Gefühl von Unverletzlichkeit die Ansteckungsgefahr enorm zu erhöhen, während auf der anderen Seite Menschen mit einem hohen Vulnerabilitätsfaktor negative Emotionalität und erhöhten Stress erleben. Diese Personengruppe neigt zur Überschätzung der Bedrohung und entwickelt eine ausgeprägte Krankheitsangst.

Das fünfte Kapitel kreist dann auch um ,kognitive Verhaltensmodelle von Krankheitsangst“. Als „Krankheitsangst“" bezeichnet der Autor die dauerhafte Neigung, die eigene Gesundheit in Gefahr zu sehen. Er sieht diese Angst auf einem Kontinuum, wobei einige Menschen zu einer übermäßigen Angst neigen, während andere sich kaum die Hände waschen, nie Distanz halten usw. Der Autor merkt an, dass ausgeprägte Krankheitsangst durch Kognitive Verhaltenstherapie sich ganz ausgezeichnet behandeln lässt.

Im sechsten Kapitel stellt der Autor „das Verhaltensimmunsystem“ vor. Das ist ein System zur Wahrnehmung und Reaktion auf Hinweisreize einer Erkrankung. Solche Hinweise erfassen Gerüche, aber auch Niesen, Husten usw. Das sind bewusste und unbewusste Hinweise auf Erkrankungen. Sie setzen Formen der Krankheitsvermeidung in Gang. Dieses Konzept scheint ausgesprochen nützlich für das Verständnis emotionaler und motivationaler Reaktionen, wenn sich Menschen von Infektionen bedroht fühlen. Das gilt besonders für gesellschaftliche Reaktionen auf Personen, von denen man annimmt, dass sie mit Infizierten in Kontakt gekommen sind. Das gilt allerdings auch als Basis für Fremdenhass und Diskriminierung. Für diese Phänomene komme die Kognitive Verhaltenstherapie ebenfalls gut in Frage. Der Autor plädiert deshalb für eine Forschung, die ermittelt, wie in Gemeinden (in Kanada?) das kollektive Verhaltensimmunsystem und die wahrgenommene Anfälligkeit für Krankheiten in bestehende soziale Programme integriert werden könnten. 
Im siebten Kapitel befasst uns der Autor mit „Verschwörungstheorien“. Dazu bemerkt er, dass sie regelmäßig in Zeiten der Unsicherheit entstehen und Menschen dazu dienen, den bedrohlichen Ereignissen einen Sinn zu verleihen. Der Autor sieht besonders in medizinischen Verschwörungstheorien die Ursache für Impfgegnerschaft und für eine generell erhöhte Pandemieangst. Er betont, dass man sich bei kommenden Pandemien besonders mit Verschwörungstheorien befassen sollte, denn sie tragen in nicht unerheblichem Ausmaß zur Ängstlichkeit in Pandemien bei.

Im achten Kapitel wendet sich Taylor „,sozialpsychologen Faktoren“ in Pandemien zu. Hier berichtet der Autor, dass sich heute in sozialen Netzwerken mit rasender Geschwindigkeit Gerüchte, Falschmeldungen und Ängste ausbreiten können. Zu dieser Frage hätten zwar schon etliche Forscher Vorschläge unterbreitet, reißerische Informationen ließen sich aber nur begrenzt einhegen. Letztlich bleibe jedem Einzelnen die Entscheidung überlassen, welche Medien er für sich als glaubwürdig betrachtet und welche nicht.

Im neunten Kapitel thematisiert der Autor „Verbesserungen der Krisen- und Risikokommunikation“ in Pandemien. Letztlich steht die Frage im Raum, wie man pandemische Phänomene so kommuniziert, dass die Kommunikation nicht kontraproduktiv wirkt. Hier wären allerdings weitere Studien notwendig, um die Effektivität von Krisen- und Risikoinformation zu verbessern.

Im zehnten Kapitel geht es um ein ,verbessertes Impfverhalten“. Beim „Impfzögern" handelt es sich um ein weitverbreitetes und sehr schwieriges Problem - sogar unter Ärzten und Pflegern. Hier scheinen immer unterschiedliche Falschmeldungen und Verschwörungstheorien eine Rolle zu spielen. Häufig finden wir auch Zweifel an der Sicherheit und Wirksamkeit von Impfungen. Außerdem spielt gar nicht selten - sogar bei medizinischem Personal - „Injektionsphobie“ eine Rolle. Für das Impfen sind immer wieder informative Kampagnen zu starten. Vielfach werden auch Zwangsimpfungen diskutiert und in manchen Ländern sogar realisiert. Es bleibt aber fraglich, ob das auf gesamtgesellschaftlicher Ebene tragfähig ist.

Im elften Kapitel thematisiert Taylor die „Behandlung emotionaler Notsituationen während einer Pandemie“. Zur Einschätzung des Ausmaßes der Verstörung einzelner Menschen zieht er den Vergleich zu Maßnahmen bei der Behandlung von Menschen bei Bombenattentaten heran, also von Menschen, die sich in extremen Notsituationen befinden. Hier haben sich seiner Meinung nach Kombinationen von Verhaltenstherapie und Medikamentengaben bewährt. Der Autor gibt allerdings zu bedenken, dass schon die Ermittlung von Vulnerabilitätsfaktoren in frühen Stadien der Pandemie sinnvoll wäre. Das könnte eine Verringerung der subjektiven Anfälligkeit für psychische Erkrankungen reduzieren.

Im zwölften und letzten Kapitel „Allgemeine Schlussfolgerungen und künftige Entwicklungen“ versucht der Autor ein Porträt der nächsten Pandemie (also unserer aktuellen Situation) zu skizieren. Dafür prognostiziert er bürgerliche Unruhen, Aufstände und Fremdenfeindlichkeit. Viele Personen werden sich nicht ans Social Distancing und andere Maßnahmen der Regierung halten. Schulen und öffentliche Einrichtungen müssen geschlossen, kulturelle Veranstaltungen lahmgelegt werden. Die Notfallambulanzen der Krankenhäuser müssen sich auf eine Welle von schwer Erkrankten einstellen. Viele Menschen werden Stressfaktoren entwickeln. Ängste und Depressionen nehmen zu. Es wird auch eine weitverbreitete Unsicherheit gras- 
sieren, ob die Pandemie wirklich vorbei ist. Anschließend betont der Autor, dass aus den vorangegangenen Ausführungen eine Vielzahl von Forschungsvorhaben zur Unterstützung in kommenden Pandemien zu extrahieren wäre.

Mein Fazit: Bei der Lektüre des Buches war ich erstaunt darüber, wie zutreffend die Aussagen des Autors über unsere aktuelle Pandemie sind. Gleichzeitig war ich betroffen angesichts der offensichtlichen Generalisierbarkeit pandemischer Ereignisse, für die man weltweit so wenig vorbereitet war und für die vor allem zu wenig und meistens zu spät Psychosoziales thematisiert wurde. So wünsche ich dem Buch einen möglichst großen Verbreitungsgrad, denn die nächste Pandemie kommt bestimmt, - und die aktuelle lässt sich vielleicht am Ende doch noch besser bewältigen als erwartet.

\section{Albert Camus: Die Pest. Reinbek: Rowohlt Taschenbuch, 90. Aufl. 2021, 368 S., $12,00 €$}

In dem Roman „Die Pest“, 1947 bei Editions Gallimard in Paris erschienen und 1949 in deutscher Übersetzung, schildert Albert Camus Vorgänge, die sich im Verlauf einer Pest-Epidemie in Oran, einer Stadt in Nordalgerien, ereignen. Die Bedeutsamkeit dieses Werkes besteht vor allem darin, dass Camus anhand der Darstellung des (fiktiven) epidemischen Prozesses und seiner Akteure seine späten philosophischen Überzeugungen verdeutlicht. Sind diese vielleicht auch in der aktuellen Pandemie relevant? Es ist jedenfalls bemerkenswert, dass dieser Roman, der einen Welterfolg erlebt hat und allein in der deutschen Übersetzung millionenfach aufgelegt worden ist, seit Beginn unserer Corona-Pandemie wiederum viel gelesen wird.

Zunächst ein kurzer Überblick der Handlung: Protagonist des Romans ist der Arzt Dr. Bernhard Rieux, 35 Jahre alt, mittelgroß mit einem energischen Kinn, immer schwarz gekleidet. Er wird als extrem pflichtbewusster Mann charakterisiert, der trotz zunehmender Erschöpfung jeweils ohne Zögern zu den hoch ansteckenden Patienten eilt. Unterstützt wird Rieux von seinem Nachbarn Tarrou, einem jungen Mann, der in seinem Tagebuch wesentliche Ereignisse des Geschehens während der Epidemie notiert. Er ist sozialpolitisch engagiert und gründet eine Schutztruppe. Ein weiterer Akteur ist Josef Grand, der als Angestellter der Stadtverwaltung alle Sterbefälle der Pest zu registrieren hat und der in seiner Freizeit versucht, sich literarisch zu verewigen. Das bleibt aber letztlich ein vergebliches Unterfangen, denn er kommt nie über den ersten Satz hinaus. Ein anderer Akteur ist Cottard, ein Vertreter und Schmuggler von Wein, der zunächst einen Selbstmord inszeniert, in Anbetracht der Pest aber anscheinend neuen Lebensmut gewinnt. Besondere Bedeutung haben zwei weitere Menschen für Rieux: Das ist zunächst Rambert, ein politisch engagierter Journalist, der nur vorübergehend nach Oran gekommen war, um über ,die Algerische Frage“ zu schreiben. Er möchte nun zu einer Frau zurückkehren, mit der er wohl eine intensive Liebesbeziehung in einer anderen Stadt aufgenommen hatte. Er leidet nämlich extrem unter seiner Einsamkeit. Da aber wegen der Epidemie alle Ein- und Ausgänge der Stadt versperrt sind, versuchte Rambert zunächst, illegale Möglichkeiten zu nutzen, um aus der Stadt zu entkommen. Erst als er Zeuge wird, mit welch bedingungsloser Hingabe Rieux Menschen zu retten versucht, erwacht auch in ihm die Bereitschaft zu helfen. So schließt er sich dem Trupp von Unterstützern an, die Tarrou ins Leben gerufen hat. Bedeutsam ist auch der Richter Othon, 
der seinen kleinen todkranken Sohn zu Rieux bringt. Leider muss aber der Kleine qualvoll sterben, obwohl er ein spezielles Serum gegen die Pest erhalten hatte. Einige Wochen später folgt ihm sein Vater in den Tod.

Ein zentraler Antagonist zu Rieux ist der Jesuitenpater Paneloux. Von ihm werden zwei Predigten berichtet: In der ersten, zu Beginn der Epidemie, spricht er in einem alttestamentarischen Verständnis von der Epidemie als Gottesstrafe. In der zweiten Predigt, als die Krise ihren Höhepunkt erreicht, spricht er Gott eher vorwurfsvoll, fast blasphemisch an. Mit ihm diskutiert Rieux ausführlich über den Sinn der lebensbedrohlichen Krankheit und konfrontiert ihn scharf mit seinen religiösen Phrasen. Diese scheinen vor allem angesichts des grausamen Sterbens von Othons kleinem Sohn obsolet. Paneloux stirbt am Ende einsam und verbittert.

Die persönlichsten Beziehungen hat Rieux zu zwei Frauen: Seine Mutter wird als liebenswürdige, besonnene Frau geschildert. In ihrer Gegenwart schöpft er jeweils neue Kraft. Die andere Frau ist seine Gattin. Da sie sich wegen einer schweren Lungenerkrankung an einem entfernten Ort in Kur begeben muss, hat sich Rieux schon vor der Epidemie von ihr verabschiedet. Er sieht sie nie wieder. Nach Beendigung der Pest erhält er lediglich eine Nachricht, dass sie während ihrer Kur verstorben ist.

Als Vorlage für die Beschreibung des Pest-Geschehens diente Camus wohl die Seuche, die etwa 100 Jahre früher in Marseille gewütet hat. Die Vorboten der Pest sind bei Camus - wie schon in der Antike - tote Ratten (aus medizinischer Sicht sind es allerdings Rattenflöhe, die auch für Menschen gefährliche Viren übertragen). Als erster sieht der Concierge Michel eine tote Ratte, woraufhin er auch als erster erkrankt. Er bekommt zunächst hohes Fieber, sodann eitrige Beulen an den Lymphknoten, die, als sie aufgeschnitten werden, sich als gelb-brauner Brei ergießen. Rieux ahnt schnell, um was es sich handelt, und benachrichtigt Dr. Richard, den Vorsitzenden der Ärzteschaft. Dieser scheut zunächst die Veröffentlichung, vor allem die Diagnose „Pest“. Als aber mehr als 20 Kranke schwarze Flecken an Bauch und Beinen aufweisen, muss er Rieux zustimmen und verfügt die Quarantäne der Kranken. Am Anfang der Epidemie handelt es sich um eine Form von Beulenpest, nach einiger Zeit mutiert sie aber zur Lungenpest, d. h. nach einem sehr hohen Fieber entwickelt sich schwere Atemnot, die zum Tode führt.

Dieses Buch nimmt in Leben und Werk des Philosophen Camus eine besondere Stellung ein, denn es weist Bezüge auf zu seiner Biographie und zu seiner späten philosophischen Orientierung (zur Biographie vgl. Radisch 2013). Camus wurde 1913 im französisch besetzten Nordalgerien als Sohn armer Leute geboren. Der Vater war französischer Landarbeiter, die Mutter mittellose Spanierin. Aufgrund seiner Intelligenz durfte er eine höhere Schule besuchen und begann danach Philosophie zu studieren. Er wollte eigentlich Lehrer werde. Da er aber als junger Mann an TBC erkrankt war, wurde er nicht als Lehrer eingestellt. So begann er, sich journalistisch zu betätigen und kritische Texte zu schreiben. Seine ersten Arbeiten waren stark von seiner Zugehörigkeit zur kommunistischen Partei und vom Freiheitskampf der Algerier gegen Frankreich beeinflusst. Im weiteren Verlauf, als Frankreich von den Nazis besetzt wurde, engagierte er sich in der Resistance. Die Niederlage Frankreichs durch Deutschland, aber auch Enttäuschungen über seine Partnerin erzeugten bei ihm zeitweise eine Stimmung von Sinnlosigkeit. Ihm schien nun jedes menschliche 
Streben absurd. In dieser Zeit schrieb er seinen berühmten Essay „Le mythe de Sisyphe“ (Camus 1942). Nach der Niederlage der Deutschen in Stalingrad hellte sich allerdings seine Stimmung wieder auf, und er begann eine fast fünfjährige Arbeit an seinem Roman „Die Pest“ (Camus 1947). Dieses Werk spiegelt den Endpunkt seiner philosophischen Entwicklung, denn hier zeigt er am Beispiel von Dr. Rieux und seinen Mitakteuren, dass es letztlich um keine politischen Positionen, um keine religiösen Überzeugungen, selbst nicht um individuelles Glück gehen kann, sondern dass im Zusammenleben von Menschen einzig und allein die Humanität, genauer, Solidarität und „menschliche Wärme“ zählen.

Diese Haltung ist aber eine Anforderung an die menschliche Reife, die - wie Camus zeigt - keineswegs von jedem erwartet werden kann. Im Gegenteil, anhand seiner Akteure begegnen uns alle Spielarten des Widerstands, die wir auch in der aktuellen pandemischen Situation wahrnehmen können. Da besteht zunächst - am Beispiel des Leiters der Ärzteschaft Dr. Richard - die Neigung, die Schwere der Krankheit und vor allem ihre epidemische Bedeutung zu verleugnen. Genau das scheint ja auch anfangs in China passiert zu sein, im weiteren Verlauf allerdings auch z. B. bei Anhängern der QAnon-Bewegung und anderen z. T. parteipolitischen Akteuren. Sodann begegnet uns in Rambert Widerstand gegen die Einsamkeit in der Quarantäne, wie sie von vielen Menschen auch heute geäußert wird. Und uns begegnet das geradezu absurd erscheinende Aufbäumen gegen Quarantänemaßnahmen bei Cottard, wie sie einige deutsche Künstler und Künstlerinnen in ihrer Verzweiflung erkennen ließen. Selbstverständlich finden wir aber auch heute tiefe menschliche Trauer über den Tod von Angehörigen wie bei Othon - und wir finden auch heute eine Vielzahl von Ärzt/innen und Pfleger/innen, die sich vorbildlich human für die Pandemiekranken aufopfern wie Dr. Rieux und Tarrou.

\section{Literatur}

Camus, A. (1942). Le mythe de Sisyphe. Paris: Gallimard. Dt.: Der Mythos des Sisyphos - ein Versuch über das Absurde (Übersetzung von Vincent von Wroblewsky). Reinbek: Rowohlt, 1999.

Camus, A. (1947). La Peste. Paris: Gallimard. Dt.: Die Pest (vom Verfasser autorisierte Übersetzung aus dem Französischen von Guido Meister). Bad Salzig: Karl Rauch, 1949. Neuausgabe: Reinbek: Rowohlt Taschenbuch, 90. Aufl. 2021

Radisch, I. (2013). Camus - Das Ideal der Einfachheit. Eine Biographie. Reinbek: Rowohlt. 